\title{
Recomposition régionale dans le Sud bolivien et migrations vers l'Argentine
}

\section{Nicolas d'Andréa}

\section{(2) OpenEdition \\ 1 Journals}

Édition électronique

URL : https://journals.openedition.org/remi/4185

DOI : $10.4000 /$ remi.4185

ISSN : $1777-5418$

Éditeur

Université de Poitiers

\section{Édition imprimée}

Date de publication : 1 octobre 2007

Pagination : 173-198

ISBN : 978-2-911627-46-6

ISSN : 0765-0752

Référence électronique

Nicolas d'Andréa, " Recomposition régionale dans le Sud bolivien et migrations vers l'Argentine », Revue européenne des migrations internationales [En ligne], vol. 23 - n² | 2007, mis en ligne le 01

octobre 2010, consulté le 15 avril 2022. URL : http://journals.openedition.org/remi/4185 ; DOI : https:// doi.org/10.4000/remi.4185

Ce document a été généré automatiquement le 15 avril 2022.

(c) Université de Poitiers 


\title{
Recomposition régionale dans le Sud bolivien et migrations vers l'Argentine
}

\author{
Nicolas d'Andréa
}

1 L'organisation de l'espace bolivien est en profonde mutation depuis le milieu du $\mathrm{XX}^{\mathrm{e}}$ siècle. Jusqu'à cette époque, l'essentiel de la population vivait en altitude, dans la partie occidentale du pays (altiplano et vallées inter-andines). Tandis que les basses vallées, (massif subandin), les plaines de l'Amazonie et du Chaco constituaient jusqu'aux frontières du Brésil, de l'Argentine et du Paraguay, un vaste espace oriental peu intégré et sous-occupé. Ce sont les politiques interventionnistes des années 1940-1950, pariant sur la mise en valeur de ces régions, qui ont ouvert la voie d'une véritable redistribution du peuplement ${ }^{1}$ (d'Andréa, 2004). Le mouvement se trouve alors amplifié par la transition démographique ${ }^{2}$ et l'accélération de la concentration urbaine. Un aspect emblématique du processus est la modification de la hiérarchie des capitales départementales. En 1950, La Paz était l'unique grande ville du pays, avec ses 320000 habitants, Santa Cruz n'en comptant alors que 43 000. En 2001, la population de La Paz était de 1437000 habitants, contre 1113000 pour Santa Cruz, qui devrait dépasser sa rivale andine en $2007^{3}$.

2 Sur le plan économique, le pays est passé d'une dépendance absolue vis-à-vis des produits miniers et des ports du Pacifique, à une certaine diversification de sa base exportatrice, grâce à l'exploitation des hydrocarbures et au développement des cultures agro-industrielles. Aujourd'hui, la réorientation des échanges vers la façade atlantique continue de s'affirmer avec deux partenaires privilégiés - l'Argentine et le Brésil-, notamment par le biais de l'intégration énergétique. En termes de différenciation régionale, ces changements bénéficient surtout au département du Santa Cruz et à sa capitale. Mais ils s'accompagnent aussi de l'essor de certaines périphéries frontalières, comme l'a déjà signalé $\mathrm{H}$. Rivière d'Arc à la fin des années 1970. C'est l'un de ces régionalismes émergents qui est analysé dans le présent article, à 
partir d'enquêtes socio-démographiques réalisées dans le département du Tarija, limitrophe de l'Argentine.

3 Ainsi, les transformations régionales durant la seconde moitié du XX $\mathrm{X}^{\mathrm{e}}$ siècle ont abouti à un nouveau positionnement du Tarija dans l'espace national et international. Devenu attractif, riche de ses hydrocarbures (l'essentiel des réserves de gaz du pays), le département accueille des flux migratoires qui nourrissent l'expansion de la frontière agraire et la croissance des villes. De nouvelles campagnes, de nouvelles échelles urbaines, l'intégration progressive du département, sa meilleure accessibilité, signalent un système régional plus complexe (Martin, 2005); d'où l'intensification et la diversification des mobilités spatiales. Or, la réalité qui perdure dans le Tarija est tout de même celle d'un pays d'émigration, en prise avec de grandes difficultés sociales qui trouvent un exutoire dans l'expatriation, en Argentine le plus souvent. Par leur proximité de la frontière, les habitants du Tarija se trouvent ainsi, plus que d'autres, confrontés à l'alternative de l'espace argentin.

4 À la question des effets des frontières sur les populations vivant à leur contact ou à leur proximité (Renard, 1992), cet article répond en observant comment se décline la migration internationale à l'échelle d'une région complexe. L'hypothèse du déterminisme frontalier confronté à la diversité des dynamiques territoriales conduit ainsi à révéler l'empreinte générale de la frontière sur la société régionale. Par ailleurs, l'article se propose d'examiner l'insertion de cette population frontalière dans le système migratoire Bolivie-Argentine, en relation avec le processus de recomposition régionale et les cycles de l'économie argentine. Comment s'articulent les migrations internes et internationales dans le Tarija ? Dans quelle mesure les courants migratoires issus du département se distinguent-ils de la tendance de localisation des Boliviens en Argentine? L'analyse met en particulier l'accent sur le lien spécifique avec les provinces argentines frontalières, illustrant la relative permanence de l'aire d'intégration des Andes centro-méridionales (Langer, 1990).

L'article caractérise tout d'abord la dynamique spatiale du courant migratoire bolivien en Argentine. Il montre notamment à partir de sources secondaires, que l'attractivité des provinces frontalières s'est progressivement réduite, exceptée peut-être, pour les habitants du Sud bolivien. L'article présente alors le département du Tarija au travers des enquêtes réalisées de 2001 à 2002 et met en évidence la diversité des dynamiques territoriales qui conditionnent le rapport spécifique des populations à l'espace argentin. Il propose enfin une observation comparée des migrations internationales dans les localités enquêtées, révélatrice du contraste des processus migratoires locaux. 


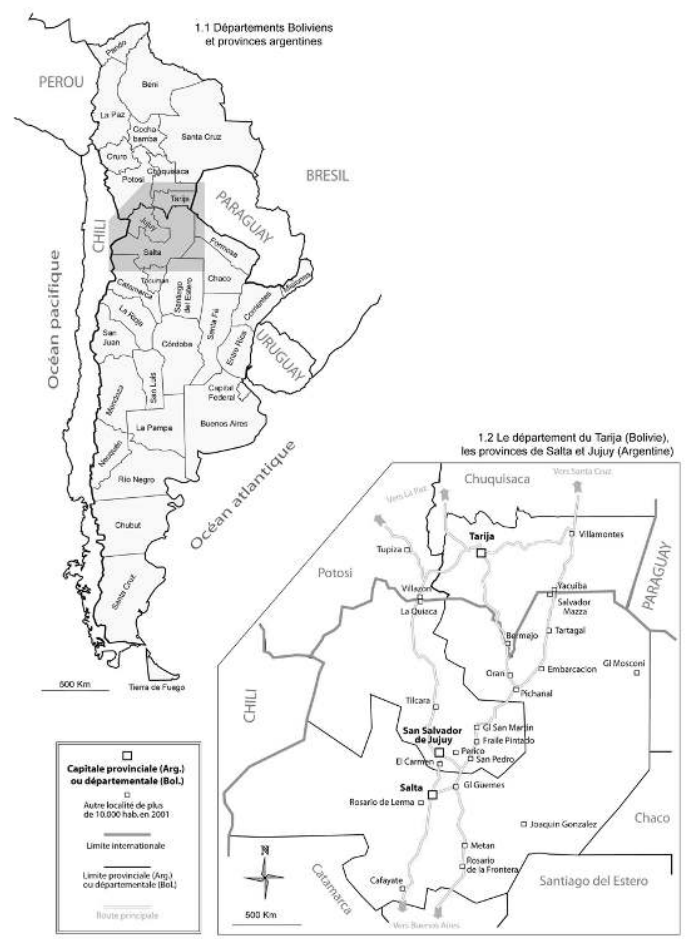

\section{L'Argentine, destination de prédilection des migrants internationaux boliviens}

6 L'Argentine est historiquement le principal pôle d'accueil des migrations internationales en Amérique du Sud. Pays d'immigration nette, grâce aux flux venus d'Europe, relayés après les années 1940 par ceux des pays proches, il est jusqu'aux années 1990 la destination principale sur le continent, des émigrés boliviens, paraguayens, chiliens et uruguayens. Les Boliviens qui migrent à l'étranger se dirigent en très forte majorité vers l'Argentine. Ce courant migratoire n'a cessé de se renforcer et de se diffuser dans l'espace argentin. Toutefois, il semble nécessaire d'interroger ces tendances dans le contexte des évolutions récentes de l'économie argentine et du système migratoire sud-américain qui se projette désormais vers l'Europe.

\section{Vers de nouvelles destinations internationales?}

Les recensements argentins montrent que le courant migratoire des Boliviens a connu un fort développement dans la seconde moitié du $\mathrm{XX}^{\mathrm{e}}$ siècle (graphique 1). On peut rattacher cette rupture, d'une part, au contexte de modernisation de la société bolivienne, qui intervient avec la Révolution Nationale au début des années 1950. Celleci engendre notamment une Réforme agraire, qui abolit les régimes de quasi-servage, imposés dans les latifundios aux populations paysannes, et libère de ce fait la mobilité de la main-d'œuvre. D'autre part, l'Argentine s'industrialisant et s'urbanisant, les migrations internes sont susceptibles d'avoir généré un besoin de main-d'œuvre de substitution dans certains secteurs d'activité traditionnels. Ainsi, l'observation 
conjointe des recensements des pays d'Amérique et des Caraïbes ${ }^{4}$ permet d'évaluer que l'Argentine concentrait $75 \%$ des natifs de Bolivie recensés ailleurs sur le continent, dans les années 1970 et 1980 . Cette proportion a diminué de $5 \%$ au profit des ÉtatsUnis, selon les recensements du début des années 1990, s'affirmant comme la seconde destination, avec $14 \%$ des natifs de Bolivie résidant à l'étranger ${ }^{5}$.

Graphique 1 : La population native de Bolivie résidant en Argentine, par recensement (effectifs et taux de variation annuel moyen)

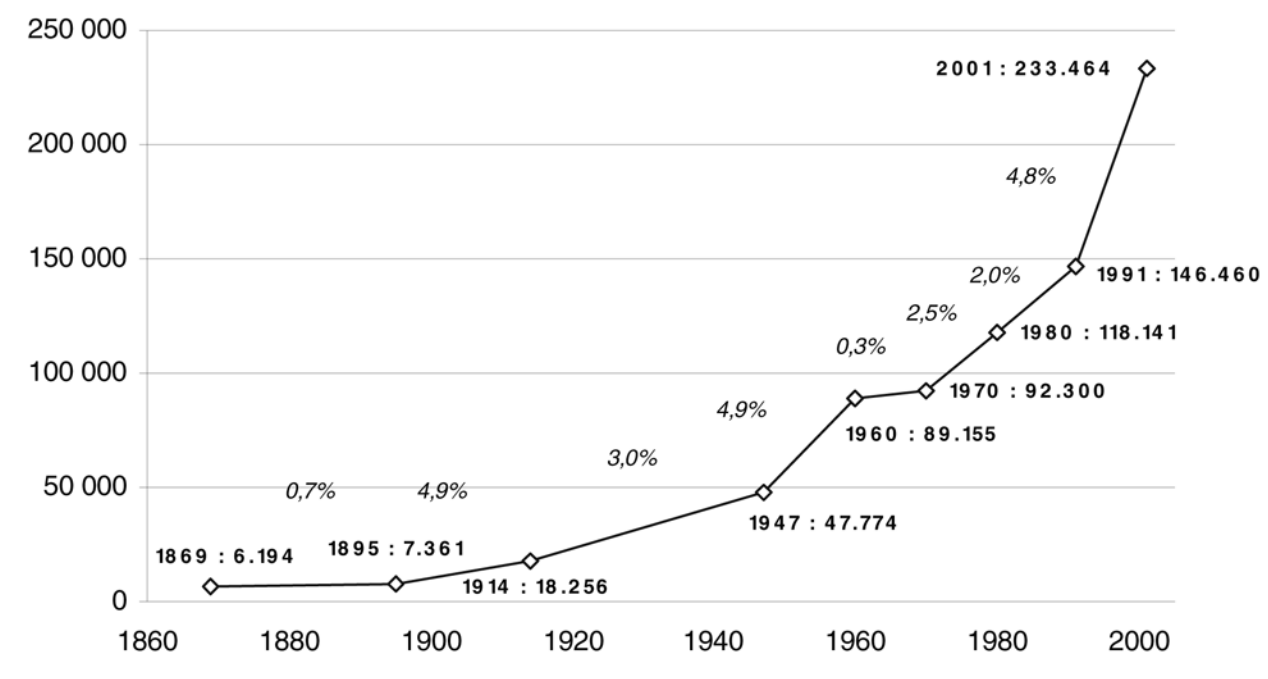

Source : INDEC

Ces faits s'inscrivent dans le contexte des vagues d'hyperinflation des années 1980 et du début des années 1990. Or, le cycle s'interrompt avec l'introduction d'une nouvelle monnaie, convertible à taux de change fixe avec le dollar: en 1992, un peso vaut désormais un dollar. À partir de ce moment, la stabilité monétaire rétablie, l'Argentine devient extrêmement attractive pour les travailleurs des pays voisins, comme en témoigne l'exceptionnelle augmentation du nombre des Boliviens recensés (graphique 1). Mais la période est éphémère, la conjoncture économique se retourne, et les conditions sociales se dégradent rapidement, en particulier dans les provinces du Nord-Ouest, frontalières de la Bolivie. Fin 2001 s'initie une série de dévaluations qui conduit à diviser la valeur du peso par trois. La crise est politique, économique, fiscale et sociale (forte hausse du chômage, baisse du pouvoir d'achat, accroissement inédit de l'émigration). Depuis, l'économie argentine réalise un redressement spectaculaire grâce aux exportations, affiche des taux de croissance à deux chiffres, sans pour autant résoudre la crise sociale, du fait de l'inflation et de l'extrême concentration des revenus (Della Paolera, 2006).

9 La succession de cette phase d'attractivité des années 1990, de la crise de 2001-2002, et de la récupération postérieure constitue une période particulièrement tumultueuse dans l'histoire du couple migratoire Bolivie-Argentine. En définitive, d'après les recensements, la présence des Boliviens en Argentine s'est nettement affirmée à l'issue de l'essor des années 1990 : ils constituent maintenant le quart des migrants provenant des pays proches, après les Paraguayens (35\%) et devant les Chiliens ( $23 \%)$. Mais les conséquences démographiques de la crise et de la phase actuelle sont difficiles à cerner. En comparant les résultats des enquêtes ménage entre 2000 et 2002, E. Bologna (2005) conclut que la crise n'a pu déboucher sur une sortie massive des immigrés ${ }^{6}$. Une source 
comme l'attribution de permis de résidence définitive (tableau 1) en signale bien l'impact, par une baisse de $40 \%$ entre 2001 et 2002. Elle témoigne aussi d'une reprise dès 2003, profitant tout particulièrement aux Boliviens, qui deviennent les premiers bénéficiaires en 2004, avec $25 \%$ de ces autorisations.

Tableau 1 : Permis de résidence définitive en Argentine délivrés entre 1999 et 2004, selon l'origine du bénéficiaire

\begin{tabular}{|l|l|l|l|l|l|l|}
\hline & 1999 & 2000 & 2001 & 2002 & 2003 & 2004 \\
\hline Bolivie & $398116 \%$ & $760923 \%$ & $580918 \%$ & $369620 \%$ & $553123 \%$ & $709936 \%$ \\
\hline Pérou & $70928 \%$ & $\begin{array}{l}11626 \\
34 \%\end{array}$ & $787125 \%$ & $437524 \%$ & $597925 \%$ & $393820 \%$ \\
\hline $\begin{array}{l}\text { autres pays } \\
\text { limitrophes }\end{array}$ & $544022 \%$ & $585117 \%$ & $710923 \%$ & $429123 \%$ & $519322 \%$ & 4020 \\
\hline autres Amérique & $317313 \%$ & $330010 \%$ & $359611 \%$ & $209111 \%$ & $234510 \%$ & $17469 \%$ \\
\hline Europe & $333913 \%$ & $28709 \%$ & $317510 \%$ & $191410 \%$ & $20879 \%$ & $15798 \%$ \\
\hline Reste du monde & $22209 \%$ & $24607 \%$ & $385612 \%$ & $217912 \%$ & $288312 \%$ & $11446 \%$ \\
\hline \hline Total & 25245 & 33716 & 31416 & 18546 & 24018 & $\begin{array}{l}19526 \\
100 \%\end{array}$ \\
\hline
\end{tabular}

Source : Direction Nationale des Migrations (DNM).

10 Ces informations suggèrent que la crise de 2001-2002 marque une fin de cycle haut dans le régime des migrations, mais réaffirment le rôle prééminent de l'espace argentin. Néanmoins, les évolutions récentes du système migratoire sud-américain sont de nature à interpeller. Car des pôles d'attraction nouveaux s'affirment dans la région (le Chili notamment), et en Europe (l'Espagne, l'Italie, le Portugal en particulier). Dans les principaux pays sud-américains expulseurs de population, l'Espagne est aujourd'hui la première destination des migrations internationales, devant les États-Unis ${ }^{7}$. Or, l'arrivée des Boliviens est récente, mais connaît un régime de progression très rapide (Domingo et Martinez 2005). En cinq ans, ils sont devenus le quatrième courant migratoire latino-américain, passant devant les Péruviens en 2005 et se rapprochant des Argentins en $2006^{8}$.

\section{La dynamique spatiale du courant migratoire en Argentine : des provinces frontalières moins attractives, mais encore fortement connectées au sud bolivien}

11 La présence des Boliviens en Argentine a longtemps été déterminée par l'économie régionale du Nord-ouest argentin. Ainsi, l'exploitation de la canne à sucre génère dès la fin du XIXe siècle un système migratoire basé sur l'emploi de main-d'œuvre agricole temporaire. La politique de développement agro-industriel des années 1950-1960 
redynamise l'attractivité des provinces de Jujuy et Salta, notamment avec la culture du tabac. Mais la mécanisation (années 1970) va progressivement diminuer le besoin de main-d'œuvre non-résidente (Whiteford, 1981), tandis que parallèlement, les Boliviens s'insèrent dans les marchés du travail de régions de plus en plus éloignées de la frontière (Benencia et Karasyk, 1995; Domenach et Celton, 1995): Buenos Aires principalement, puis les grands bassins viticoles ou fruticoles (Mendoza en particulier), et les principales villes avec leurs périphéries maraîchères, jusqu'aux ports du Sud. Cette expansion du courant migratoire aboutit à une nouvelle distribution spatiale, selon laquelle l'importance des provinces frontalières ne cesse de décliner.

Le dernier recensement indique d'ailleurs une accélération de cette tendance : Salta et Jujuy concentraient $41,1 \%$ des natifs de Bolivie en 1980, 34,5\% en 1991 et seulement $22,3 \%$ en 2001. Il faut toutefois noter que cette évolution est plus fortement imputable à Jujuy (20,1\% des natifs en 1991, 12,3\% en 2001). Entre 1991 et 2001, d'après des effectifs censitaires qui reflètent au mieux la migration légale, la présence des Boliviens a diminué de $5 \%$ dans la province de Jujuy, a augmenté de $8 \%$ dans la province de Salta, de $81 \%$ dans la province de Buenos Aires et de $88 \%$ dans le reste du Pays. La structure d'âges des Boliviens répartie par province corrobore bien ces tendances (tableau 2). La moindre attractivité actuelle des provinces frontalières, Jujuy en particulier, se traduit par un vieillissement avancé: les provinces frontalières concentrent $22 \%$ des Boliviens mais $53 \%$ des plus de 65 ans. Tandis que les Boliviens de Buenos Aires et des autres provinces sont surreprésentés dans toutes les classes d'âge inférieur à 50 ans.

Tableau 2 : La population native de Bolivie résidant en Argentine en 2001, selon l'âge et la province

\begin{tabular}{|c|c|c|c|c|c|c|c|c|c|c|c|c|c|c|c|}
\hline \multirow{2}{*}{$\begin{array}{|ll|}\text { âge } & \text { province } \\
\end{array}$} & \multicolumn{3}{|c|}{ Jujuy } & \multicolumn{3}{|c|}{ Salta } & \multicolumn{3}{|c|}{ Buenos Aires } & \multicolumn{3}{|c|}{ Autres provinces } & \multicolumn{3}{|c|}{ Total } \\
\hline & eff. & $\%$ col. & oli. & eff. & $\%$ col. & $\%$ li. & eff. & col. & $\%$ li. & eff. & $6 \mathrm{col}$. & $\%$ li. & eff. & $\%$ col. & $\%$ li. \\
\hline 0.14 ans & 930 & 3,2 & 4,9 & 1151 & 4 & 6 , & 262 & 3 & & 0 & 9,5 & 45 & 26 & 8,2 & 00,0 \\
\hline $15-24$ ans & 2623 & 9,1 & 6,8 & 2917 & 12,5 & 7,6 & 15336 & 17,2 & 39,9 & 17567 & 19,1 & 45,7 & 38443 & 16,5 & 00,0 \\
\hline $25-49$ ans & 10162 & 35,4 & 8,7 & 9712 & 7 & 8,4 & 46861 & 52,5 & 40,3 & 49574 & 8 & 42,6 & 116309 & 49,8 & 100,0 \\
\hline $50-64$ ans & 8282 & 28,8 & 21,2 & 5431 & 23,3 & 13,9 & 13899 & 15,6 & 35,6 & 11451 & 12,4 & 29,3 & 39063 & 16,7 & 100,0 \\
\hline 55 ans & 6738 & & 32,8 & 4081 & & 19,9 & 4948 & & 24,1 & & & 23,2 & 20523 & 8,8 & 00, \\
\hline otal & 28735 & 00,0 & 12,3 & 23292 & 00,0 & 10,0 & 89306 & 00,0 & 38,3 & 92131 & 100,0 & 39,5 & 233464 & 100,0 & 100, \\
\hline
\end{tabular}

Source : INDEC, recensement 2001.

13 Ainsi, les provinces frontalières ont été le premier territoire d'installation de générations de migrants boliviens. L'enquête complémentaire sur les migrations internationales (ECMI) ${ }^{9}$ livre des éléments d'analyse concernant les Boliviens résidant dans les agglomérations de Jujuy, Salta et Buenos Aires. Tout d'abord, une illustration partielle dans le cas du Grand Buenos Aires ${ }^{10}: 17 \%$ des Boliviens arrivés avant 1970 ont d'abord résidé dans la province de Jujuy ; $5 \%$ de ceux arrivés entre 1970 et 1979 et moins de $1 \%$ pour les cohortes d'arrivées les plus récentes. Ensuite, l'enquête de Salta signale aussi la baisse d'attractivité de Jujuy, puisque $26 \%$ des résidents Boliviens arrivés avant 1970 y avaient d'abord résidé, contre $2 \%$ pour la cohorte 1990-2002. Enfin, l'ECMI suggère que le rôle des provinces frontalières s'est atténué avec la simplification des parcours migratoires. Avant les migrants avaient une trajectoire migratoire plus complexe ${ }^{11}$ et les provinces frontalières jouaient fréquemment le rôle d'étape initiale en Argentine.

14 Cependant, si l'attractivité des provinces frontalières s'est atténuée à l'échelle du courant migratoire, dans certaines régions de Bolivie, cette tendance n'est pas forcément dominante. Car les liens historiques entre la Bolivie méridionale et le Nord- 
ouest argentin, ainsi que l'avantage de la proximité, sont susceptibles d'entretenir localement un schéma plus traditionnel. L'analyse d'un échantillon des registres de la Direction Nationale des Migrations (Benencia et Karasyk, 1999) avait permis de signaler, dans la province de Jujuy, un renforcement de la présence des Boliviens originaires des départements limitrophes (Potosi et Tarija). Plus récemment, l'enquête ECMI (tableau 3) montre le contraste des origines régionales: à Buenos Aires, les migrants provenant du Cochabamba constituent le champ migratoire dominant; alors que dans les deux agglomérations frontalières, les migrants des départements voisins (Potosi et Tarija) sont les plus représentés, quelle que soit la période d'installation.

Tableau 3 : La population native de Bolivie résidant en Argentine en 2001, selon le dernier département de résidence en Bolivie et la période d'installation en Argentine, par localité

\begin{tabular}{|c|c|c|c|c|c|c|c|c|c|c|c|}
\hline 冚 & $\begin{array}{l}\text { Dernier } \\
\text { département de } \\
\text { résidence en } \\
\text { Bolivie } \\
\end{array}$ & avant & 1970 & 1970 & 1979 & 1980 & 1989 & 1990 - & 2003 & eff. & $\%$ \\
\hline \multirow{7}{*}{ 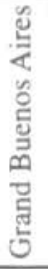 } & Cochabamba & 4753 & $32 \%$ & 5330 & $48 \%$ & 7196 & $52 \%$ & 8101 & $38 \%$ & 25380 & $42 \%$ \\
\hline & Potosí & 4779 & $33 \%$ & 2385 & $22 \%$ & 2704 & $20 \%$ & 3478 & $16 \%$ & 13346 & $22 \%$ \\
\hline & $\mathrm{La} \mathrm{Paz}$ & 2458 & $17 \%$ & 1192 & $11 \%$ & 1591 & $12 \%$ & 4279 & $20 \%$ & 9520 & $16 \%$ \\
\hline & Santa Cruz & 344 & $2 \%$ & 764 & $7 \%$ & 284 & $2 \%$ & 1587 & $7 \%$ & 2979 & $5 \%$ \\
\hline & Reste & 2288 & $16 \%$ & 1344 & $12 \%$ & 1855 & $14 \%$ & 3695 & $17 \%$ & 9182 & $15 \%$ \\
\hline & Non rense & 13 & $0 \%$ & 67 & $1 \%$ & 99 & $1 \%$ & 136 & $1 \%$ & 315 & $1 \%$ \\
\hline & Total & 14635 & $100 \%$ & 11082 & $100 \%$ & 13729 & $100 \%$ & 21276 & $100 \%$ & 60722 & $100 \%$ \\
\hline \multirow{7}{*}{ 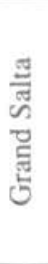 } & Potosi & 312 & 3 & 568 & $31 \%$ & 972 & 310 & 572 & $37 \%$ & 3424 & $32 \%$ \\
\hline & Tar & 1099 & $26 \%$ & 378 & $21 \%$ & 939 & $30 \%$ & 260 & $17 \%$ & 2676 & $25 \%$ \\
\hline & Cochabamb & 688 & $16 \%$ & 258 & $14 \%$ & 260 & $8 \%$ & 214 & $14 \%$ & 1420 & $13 \%$ \\
\hline & $\mathrm{LaPaz}$ & 228 & $5 \%$ & 182 & $10 \%$ & 180 & $6 \%$ & 10 & $1 \%$ & 600 & $6 \%$ \\
\hline & Reste & 872 & $21 \%$ & 434 & $24 \%$ & 765 & $24 \%$ & 496 & $32 \%$ & 2567 & $24 \%$ \\
\hline & Non rense & 43 & $1 \%$ & - & - & 12 & $0 \%$ & - & $\cdot$ & 55 & $1 \%$ \\
\hline & Total & 4242 & $100 \%$ & 1820 & $100 \%$ & 3128 & $100 \%$ & 1552 & $100 \%$ & 10742 & $100 \%$ \\
\hline \multirow{7}{*}{ 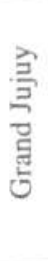 } & Poto & 1070 & 3 & 7 & $43 \%$ & 1167 & $49 \%$ & 458 & $55 \%$ & 4249 & $41 \%$ \\
\hline & $\mathrm{T}$ arija & 1431 & $27 \%$ & 549 & $31 \%$ & 644 & $27 \%$ & 174 & $21 \%$ & 2798 & $27 \%$ \\
\hline & Cochabamba & 856 & $16 \%$ & 89 & $5 \%$ & 128 & $5 \%$ & 41 & $5 \%$ & 1114 & $11 \%$ \\
\hline & $\mathrm{LaPaz}$ & 104 & $2 \%$ & 59 & $3 \%$ & 99 & $4 \%$ & 49 & $6 \%$ & 311 & $3 \%$ \\
\hline & Reste & 1012 & $19 \%$ & 297 & $17 \%$ & 330 & $14 \%$ & 104 & $13 \%$ & 1743 & $17 \%$ \\
\hline & Non renseigné & 44 & $1 \%$ & 28 & $2 \%$ & 9 & $0 \%$ & - & & 81 & $1 \%$ \\
\hline & Total & 5305 & $100 \%$ & 1788 & $100 \%$ & 2377 & $100 \%$ & 826 & $100 \%$ & 10296 & $100 \%$ \\
\hline
\end{tabular}

Source : INDEC - ECMI 2003.

\section{Le Tarija, département frontalier au cœur des transformations du Sud bolivien}

Entre $20^{\circ} 50^{\prime}$ et $22^{\circ} 50^{\prime}$ de latitude Sud, $62^{\circ} 15^{\prime}$ et $65^{\circ} 20^{\prime}$ de longitude Ouest, le plus petit département bolivien $\left(37,623 \mathrm{~km}^{2}\right)$ présente une grande variété d'écosystèmes et de paysages. À $500 \mathrm{~km}$ au nord du tropique du Capricorne, le Tarija s'étend sur une série de plissements longitudinaux qui déclinent en altitude, des sommets de la Cordillère royale (4 600 m maximum), aux derniers chaînons du massif subandin (entre 1500 et $500 \mathrm{~m}$ ), débouchant sur la plaine du Chaco (entre 300 et $500 \mathrm{~m}$ ). Le département est ainsi positionné sur les deux grandes unités qui partagent l'espace bolivien: côté occidental, s'étend un secteur de haute et moyenne montagne à tendance semi-aride, de régime thermique tempéré ; côté oriental, le massif subandin et la plaine du Chaco 
sont soumis à des températures et des précipitations plus élevées ${ }^{12}$. Le peuplement de l'espace occidental se concentre dans un système de vallées d'altitude intermédiaire (entre 1500 et $2000 \mathrm{~m}$ ), que nous désignerons du terme "vallées andines ». Celui de l'espace oriental s'inscrit dans les vallées subandines et sur le piémont du Chaco. C'est dans ces subrégions que se localisent les principales zones d'agriculture intensive et les villes.

Carte 2 : Le département du Tarija

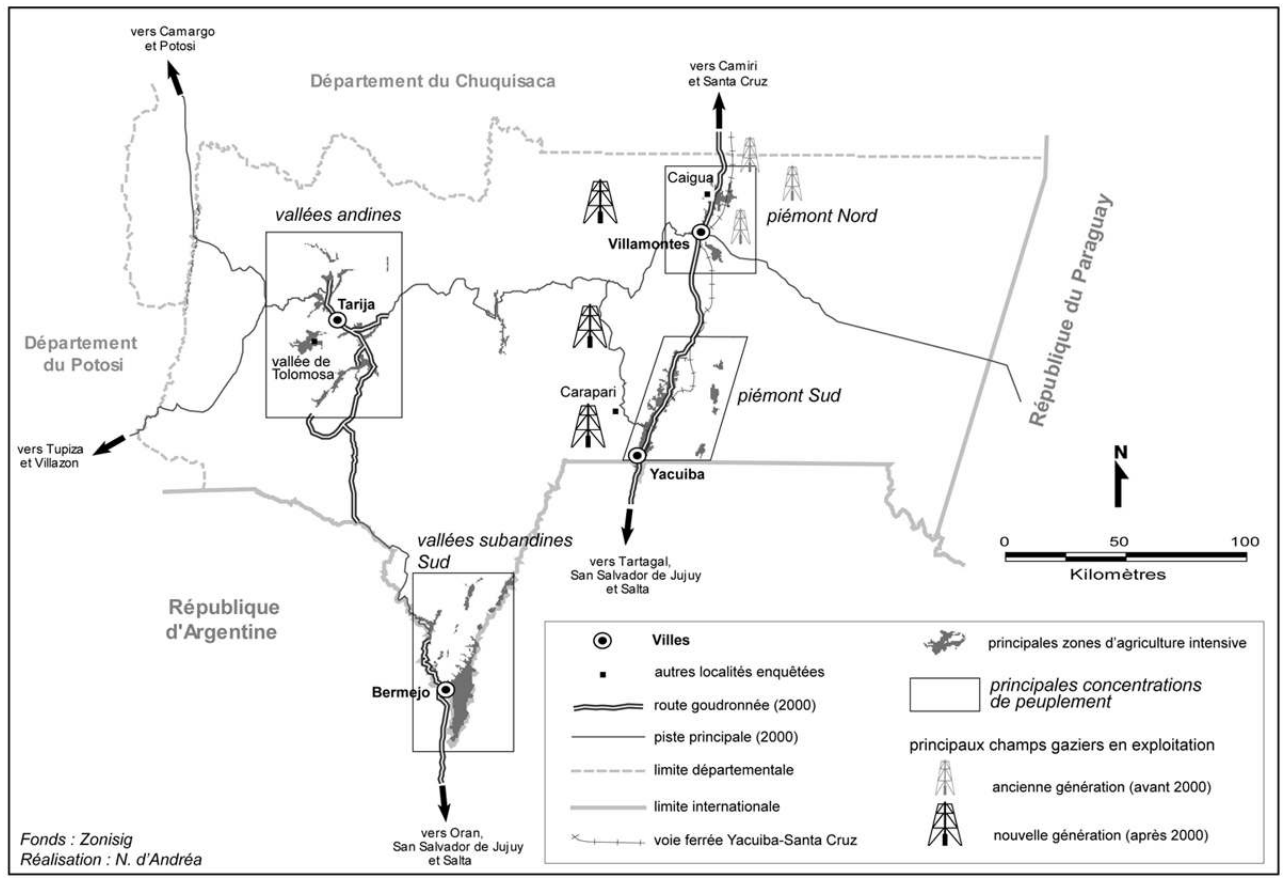

Fin de l'isolement et recomposition régionale

Les vallées du bassin-versant de la rivière Tarija constituent le cœur historique du peuplement régional : le pays des chapacos ${ }^{13}$. L'espace s'est élargi progressivement vers l'Est, frontière sauvage, pacifiée à la fin du XIX siècle. Sous l'Empire espagnol, la relative prospérité de la région est liée à son positionnement proche du corridor de transit qui relie par l'altiplano, l'estuaire de la Plata et les grandes villes de la ViceRoyauté du Pérou ${ }^{14}$ (Potosi, Lima). Sous la République (à partir de 1825), le petit département du Tarija est défavorisé par l'hégémonie des cités du Nord et leurs bassins miniers, dont l'intégration ferroviaire avec les ports du Pacifique (fin XIX ${ }^{\mathrm{e}}$ et début XX siècle) achève de marginaliser les autres régions boliviennes (Roca, 1999). Tandis que de l'autre côté de la frontière, les provinces du Nord-ouest, dont dépend aussi l'économie tarijénienne, deviennent périphériques dans l'espace argentin (J. Piel, 1989). À ce contexte de confinement s'ajoute un défaut d'intégration interne : les vallées andines et la capitale - Tarija - se trouvent mal reliées aux terres hautes et donc au principal axe de circulation transfrontalière ${ }^{15}$; elles communiquent par ailleurs faiblement avec les subrégions orientales, qui se développent en relative autonomie.

Ainsi, le département du Tarija franchit le milieu du XX $X^{e}$ siècle en état de sous urbanisation et de relatif retard économique (Schoop, 1986). Mais la région va connaître dès lors, grâce à son domaine oriental, un cycle durable d'intégration à 
l'économie nationale. Car premièrement, l'essor de la région de Santa Cruz entraine la formation d'un axe géo-commercial nouveau, qui relie la capitale orientale à l'Argentine, et assoit la position de Yacuiba (piémont du Chaco) comme principal point de transit international dans le pays ${ }^{16}$. Deuxièmement, la politique d'industrialisation nationale permet de dynamiser les fronts pionniers, notamment dans le Sud subandin, par l'introduction de la canne à sucre et sa transformation. D'où la formation d'une seconde ville-frontière - Bermejo - et la connection de celle-ci à la capitale départementale, qui engendre la structuration d'un nouveau couloir de circulation transfrontalière. Troisièmement, le département bénéficie de la rente des hydrocarbures, ce qui lui permet de moderniser son réseau routier, accentuant l'écart de développement avec les départements non producteurs. Or, ce processus connaît une impulsion inédite depuis la fin des années 1990 et la découverte de grands gisements de gaz, qui placent le Tarija au cœur des enjeux de l'intégration énergétique du Cône Sud.

\section{Une série d'enquêtes socio-démographiques en milieu urbain et rural, en zone occidentale et orientale}

18 En l'espace d'un demi-siècle, la population départementale a augmenté de 264000 individus (391 226 habitants recensés en 2001), à un rythme supérieur à la moyenne nationale ${ }^{17}$. La concentration urbaine s'est affirmée à Tarija, mais aussi grâce à l'émergence des villes-frontière (Yacuiba et Bermejo), qui ont de surcroît connu un cycle exceptionnel de prospérité commerciale et d'expansion démographique durant les années 1990, au moment de la parité peso argentin dollar (1992-2001). Par ailleurs, sur le piémont du Chaco, au centre des principaux gisements d'hydrocarbures, la localité de Villamontes s'est constituée en gros bourg, achevant la formation d'un réseau urbain régional semi-polarisé, assez original dans l'espace bolivien. Dans la nouvelle configuration du peuplement, la vitalité des campagnes andines est menacée par l'émigration, mais la primauté du cœur historique demeure, grâce à la croissance de la capitale. Côté oriental, trois sub-régions se sont consolidées, associant un centre urbain et un nouveau bassin d'agriculture intensive, résultant de l'expansion de la frontière agraire : le «triangle» de Bermejo dans la zone subandine méridionale, les piémonts Nord (Villamontes) et Sud (Yacuiba).

19 Dans ce contexte de recomposition régionale, quelles dynamiques migratoires sont à l'œuvre et quelles relations s'établissent avec l'espace argentin ? C'est pour répondre entre autres à ce questionnement, qu'un dispositif d'enquête multi-site nommé «Frontarbol» a été mis en place dans le cadre d'une coopération entre l'IRD, les universités de Cordoba (Argentine) et de Tarija. Ainsi, quatre opérations de collecte ont été menées dans le département du Tarija entre septembre 2001 et juillet 2002 concernant six unités d'observation : les villes de Tarija, Yacuiba et Villamontes, ainsi que des localités rurales positionnées dans leur périphérie - respectivement, la vallée de Tolomosa, Carapari et Caigua ${ }^{18}$. Chaque enquête a porté sur un échantillon de ménages et référence divers types de mobilités spatiales. Pour appréhender les tendances de la migration internationale dans le Tarija, le présent article se réfère principalement à deux sous-populations. Les «migrations» résultent de l'enregistrement des trois dernières migrations ayant entraîné un déplacement résidentiel d'au moins six mois, appliqué aux membres du ménage âgés de 15 ans ou plus. L'autre indicateur de la migration est la descendance des ménages : un enfant 
résidant à l'étranger est assimilé à un migrant international. Il y a migration avec retour dans le premier cas, et migration en cours dans le second.

\section{Des dynamiques territoriales singulières}

20 À la dichotomie de l'espace correspond une opposition des tendances: l'Occidente du Tarija évolue entre déprise rurale et résilience du peuplement, grâce à la croissance de la capitale; tandis que l'Oriente a connu des cycles d'expansion récents, générateurs d'accumulation mais aussi d'instabilité démographique.

\section{La zone occidentale (vallée de Tolomosa et Tarija) : entre déprise rurale et résilience du peuplement}

21 La vallée de Tolomosa regroupe onze communautés (7 328 habitants en 2001) situées entre 1850 et 2100 mètres d'altitude, à proximité de Tarija. Il s'agit d'une zone typique des vallées andines, qui a bénéficié de la réforme agraire de 1952 avec retard, les attributions de terres ayant été réglées dans les années 1970. Avec des lots relativement petits au départ, la propriété s'est rapidement fractionnée. L'enquête indique en moyenne moins de 0,5 ha cultivés par actif déclarant avoir travaillé dans l'agriculture les sept derniers jours. Le système productif est basé sur l'agriculture avec des variations selon les communautés, car les finages diffèrent par le système de culture et l'importance de l'élevage. Outre le problème de la disponibilité foncière, cette petite région est exemplaire des difficultés de l'agriculture andine : défaut d'infrastructure de stockage, érosion des sols, difficultés d'accès au marché, faible recours à l'irrigation, concurrence des produits de l'agriculture commerciale... Ce qui entraîne à des degrés divers le sous-emploi et l'émigration. En effet, le peuplement est ancien et ne se renouvelle pas, faute d'attractivité ( $91 \%$ des chefs de ménage sont natifs de la vallée, et leurs parents dans la même proportion). Du fait du départ précoce des jeunes en âge actif, la population vieillit plus rapidement. De plus, la féminisation récente de l'émigration et la limitation du potentiel de reproduction qu'elle implique accélèrent le processus. Au début des années 2000 , la vallée est engagée sur la voie de la déprise démographique.

À quelques kilomètres, Tarija constitue le grand facteur de résilience du peuplement occidental, comme les autres capitales départementales ancrées dans les vallées andines (Sucre, Cochabamba). Les habitants de Tarija ne sont pas à proprement parler des frontaliers. À 1800 mètres d'altitude, les 176 kilomètres sinueux qui séparent la ville du poste frontière de Bermejo ont longtemps constitué une distance conséquente dans l'absolu (environ 7 heures de transport en commun), mais intermédiaire à l'échelle bolivienne ${ }^{19}$. Bien que relativement isolée de l'Argentine et des grandes villes boliviennes, Tarija fait partie des capitales dont la croissance a été particulièrement soutenue durant le dernier quart de siècle. Elle a ainsi dépassé les 135000 habitants en 2001, devançant Potosi pour la première fois de l'histoire. Ce dynamisme, au-delà de l'influence de la transition démographique, est alimenté par l'importance de l'immigration et la diversification de ses foyers d'origine ${ }^{20}$ ( $68 \%$ des chefs de ménage ne sont pas natifs de la ville). Car dans le système urbain du Sud bolivien, Tarija offre un climat agréable, des fonctions administratives centrales et des services moteurs (santé, éducation, énergie, commerce) alimentant l'emploi, bref des éléments de qualité de vie 
qui la rendent attractive. Dans le contexte de la crise andine et de l'instabilité des territoires neufs de la zone orientale, c'est une "ville-refuge", au centre de circulations migratoires diverses (D’Andréa et Martin, 2007).

\section{L'Oriente et ses cycles d'expansion : colonisation agricole (Caigua), essor du gaz (Villamontes, Carapari), cycle de prospérité du commerce transfrontalier (Yacuiba)}

23 La vitalité démographique du département repose sur le nouveau dimensionnement de la capitale et sur un nombre réduit de zones de croissances à l'Est. Ces dernières ont en commun de réagir à des conjonctures qui entraînent localement des effets démographiques virulents et partiellement réversibles.

\section{Le piémont du Chaco : déboires de la filière agro-industrielle et colonisation spontanée}

24 Le piémont du Chaco est le principal foyer d'expansion de la frontière agraire et du peuplement avec le Sud de la zone subandine (Bermejo). La recomposition des structures agraires intervient dans une région traditionnellement dévolue à l'élevage bovin extensif, dominé par les grands propriétaires terriens. Comme à Bermejo, les pouvoirs publics sont à l'initiative d'un grand projet de développement: dans les années 1970, Villamontes est choisie pour devenir un pôle agro-industriel national dans le domaine des oléagineux. Une raffinerie est achevée en 1974. En sous capacité permanente, l'usine interrompt son activité à la fin des années 1990. Parmi les explications de cet échec, sont couramment évoqués (CEDLA, 1998) le défaut de régularisation des titres de propriété octroyés aux paysans, l'endettement de ces derniers, une monoculture imposée ne correspondant pas à leurs besoins alimentaires et des revenus générés insuffisants ${ }^{21}$.

C'est alors un autre modèle d'agriculture qui va spontanément se répandre, par la mise en valeur de petites parcelles du piémont, en irriguant des secteurs jusque-là délaissés ou cultivés en sec. L'enquête de la communauté de Caigua fournit un exemple de ce type d'adaptation. Le modèle de développement de cette communauté de cultivateurs aura connu deux moments principaux. Au cours d'une phase "créatrice», une population d'immigrants venus des vallées voisines a renouvelé l'usage d'un terroir sous-valorisé, en introduisant des méthodes d'irrigation permettant de pratiquer une polyculture de base maraîchère (années 1970-1990) : c'est alors le processus productif qui est déterminé par la pression démographique. Avec le succès de l'entreprise, les immigrants s'accumulent, la population augmente, la superficie des exploitations diminue pour les derniers arrivants et les mécanismes d'ajustement démographique se renforcent, définissant l'avènement d'une phase « malthusienne » : c'est maintenant la population qui doit s'adapter à la capacité productive du système agraire ${ }^{22}$.

\section{Le cycle de prospérité du commerce transfrontalier}

Durant les années 1990, la frontière boliviano-argentine a été le théâtre d'une phase de croissance urbaine exceptionnelle, due à l'essor du commerce transfrontalier. Positionnée sur le principal axe de circulation entre la Bolivie et l'Argentine, Yacuiba a naturellement été la principale bénéficiaire d'une conjoncture extrêmement favorable 
au commerce bolivien. Trois facteurs ont primé dans le processus. Premièrement, la fluctuation des taux de change. En effet, tandis qu'à partir de janvier 1992 la politique monétaire argentine fixe la parité peso-dollar pour juguler l'inflation, le boliviano continue de se déprécier et la valeur réelle du peso par rapport au boliviano augmente de $75 \%$ entre 1990 et 1994 . Deuxièmement, l'économie de la drogue ${ }^{23}$ a stimulé le commerce transfrontalier, la forme la plus commune et directe de faire du blanchiment d'argent en Bolivie étant l'importation de biens par contrebande ${ }^{24}$. Troisièmement, la permissivité des douanes des deux pays a constitué une condition favorable au système. Côté bolivien, le peu d'effectivité de la taxation des importations et le niveau des charges acquittées par les commerçants abaissent le coût des produits, sans compter la prolifération des contrefaçons. Côté argentin, les autorités ont laissé se développer la vogue du tourisme d'achat, alimentée par les transporteurs des grandes villes du Centre et du Nord, et leur clientèle de petits commerçants et de particuliers.

Inversement, c'est à l'altération de ces mêmes mécanismes qu'il faut imputer la fin du cycle. D'une part, la réduction du narcotrafic au milieu des années 1990 et la réaffirmation de la lutte contre la contrebande de part et d'autre de la frontière (1999), ont constitué des facteurs de ralentissement de l'activité commerciale. D'autre part, le basculement du taux de change avec la dévaluation argentine fin 2001 a clairement interrompu le cycle de prospérité. Le bilan de cette période exceptionnelle transparaît dans l'évolution de la population: entre 1992 et 2001, Yacuiba a connu un taux d'accroissement annuel moyen de $7 \%$, passant de 34505 à 64611 habitants $\left(12^{\mathrm{e}}\right.$ rang national). Ce chiffre serait nettement en deçà du niveau atteint au plus fort de l'essor économique. L'observation rétrospective des installations et l'identification des flux de redistribution de la population de Yacuiba vers Tarija et Villamontes placent le point d'inflexion entre 1995 et 1996. Au moment des enquêtes (2001-2002), Yacuiba était encore en phase de dépopulation.

\section{L'essor du gaz}

28 Le pétrole est exploité depuis les années 1920 en Bolivie et les premiers puits d'importance se situaient dans le Tarija. Mais ce sont les gisements du Santa Cruz qui permettent d'atteindre un niveau de production exportable en 1955. La loi prévoyant une redistribution des redevances pétrolières aux départements producteurs ${ }^{25}$, le Santa Cruz a bénéficié dès lors d'une manne fiscale décisive dans son ascension. À l'ère du pétrole, ressource finalement peu abondante, succède celle du gaz, marquée par le contrat de vente à l'Argentine (1972). Celui-ci bénéficie cette fois encore au Santa Cruz principalement, mais le Tarija commence à percevoir des niveaux de redevance inédits (plus de 3 millions de dollars annuels). À partir des années 1980, la rente des hydrocarbures est mieux répartie entre les départements producteurs, mais la production de gaz diminue. Un contrat d'exportation avec le Brésil (1991) relance l'activité et les besoins d'exploration. L'État y parvient en capitalisant l'entreprise publique Yacimientos Petroleos Fiscales Bolivianos (YPFB) en 1996. De grandes compagnies investissent (Total, Petrobras), pour des résultats inespérés : dès 1999 le niveau des réserves de gaz fait un bond historique, la Bolivie recèle désormais les plus grandes réserves d'Amérique du Sud après le Venezuela. Or, l'essentiel de cette ressource ${ }^{26}$ se localise dans le Tarija.

Ces découvertes ont des conséquences locales, nationales et internationales (Martin 2005). Pour le Tarija, dès la mise en service des nouveaux puits, les redevances 
augmentent vertigineusement : elles dépassaient les 25 millions de dollars en 2003 et le double en 2004. Au-delà de la différenciation régionale qui va s'affirmer, l'organisation spatiale du département pourrait connaître de nouvelles évolutions. L'essentiel du gaz extrait jusqu'à présent provenait de la plaine du Chaco, près de Villamontes, tandis que les nouveaux gisements se situent principalement dans les vallées subandines proches, à peine peuplées. Entre l'activité générée directement durant les phases d'exploration et de mise en œuvre de l'exploitation, d'un côté, et la redistribution d'une partie des redevances aux municipes, de l'autre, un fort impact sur le peuplement est attendu. Sans compter les perspectives d'investissements productifs (irrigation, industrialisation du gaz) qui pourraient diversifier les pôles d'attraction. Au début des années 2000, deux centres de municipe connaissent dans ce contexte une mutation rapide : Villamontes et Carapari.

Villamontes, ville de garnison, carrefour entre Tarija et Santa Cruz, centre d'un bassin d'agriculture intensive, mais aussi d'élevage bovin, se trouve à proximité des anciens et des nouveaux champs de gaz. Sa population a augmenté rapidement $(+4,0 \%$ par an de 1976 à $2001,+4,1 \%$ de 1992 à 2001), pour atteindre 16113 habitants en 2001. Les migrations ont une forte incidence sur le peuplement: les deux tiers des chefs de ménage ne sont pas natifs de la localité, plus de la moitié de la population a moins de vingt ans. Dans la zone subandine, Carapari constitue une concentration de population plus modeste (environ 1000 habitants), en voie d'urbanisation. Sa dimension de petite centralité subrégionale s'affirme (école, internat, services de santé). Ainsi, selon l'enquête, l'agriculture ne concerne plus que $13 \%$ des actifs occupés alors que $70 \%$ se classent dans les services. La croissance démographique a été stimulée par des installations récentes qui se corrèlent avec l'exploitation des gisements pétro-gaziers voisins (San Alberto). De fait, la population compte maintenant une majorité de chefs de ménages issus d'autres territoires.

\section{Contrastes et homogénéité des champs migratoires}

Dans ce contexte de recomposition régionale, comment s'exprime l'effet de frontière induit par l'accessibilité de l'espace argentin? Dans quelle mesure l'émigration locale correspond-elle aux grandes tendances de développement du courant migratoire bolivien, observées depuis l'Argentine? Notamment, comment se redéfinit la relation avec les provinces de Salta et Jujuy? Les enquêtes du programme Frontarbol apportent des éléments de réponse à partir d'indicateurs comparés qui donnent un aperçu des contrastes et de l'homogénéité des champs migratoires.

\section{L'omniprésence de la migration internationale}

Les enquêtes signalent l'omniprésence de la migration internationale, en zone occidentale et orientale, dans les villes et les campagnes (tableau 4). Le poids du phénomène, exprimé en part des ménages concernés, révèle toutefois des écarts importants. De $8 \%$ (Villa montes) à $73 \%$ (Vallée de Tolomosa) des ménages ont une expérience de la migration internationale : qu'un membre du ménage ait déjà effectué une migration, qu'un enfant du ménage réside à l'étranger ou bien que ces deux conditions soient réunies ${ }^{27}$. L'importance des prélèvements de l'émigration internationale transparaît plus nettement sur la base des ménages dont au moins un 
enfant a décohabité. Au-delà du phénomène d'exode constaté dans la vallée de Tolomosa, les ménages de Tarija et Yacuiba témoignent de fréquence des départs vers l'étranger en milieu urbain: les deux villes principales et les campagnes andines constituent ainsi les foyers majeurs de l'émigration internationale.

Tableau 4 : Fréquence de la migration internationale dans les ménages, par lieu d'enquête

\begin{tabular}{|l|l|l|l|l|l|l|}
\hline Part des ménages (en \% ) & Tarija & $\begin{array}{l}\text { Vallée de } \\
\text { Tolomosa }\end{array}$ & Yacuiba & $\begin{array}{l}\text { Villa- } \\
\text { montes }\end{array}$ & Carapari & Caigua \\
\hline $\begin{array}{l}\text { Avec enfant résidant à } \\
\text { l'étranger }\end{array}$ & 9,8 & 44,8 & 9,1 & 4,1 & 3,0 & 6,9 \\
\hline $\begin{array}{l}\text { Avec enfant résidant à } \\
\text { l'étranger (1) }\end{array}$ & 29,4 & 78,3 & 24,1 & 9,8 & 6,3 & 19,2 \\
\hline $\begin{array}{l}\text { Avec individu ayant effectué } \\
\text { une MI }\end{array}$ & 13,4 & 45,8 & 14,4 & 4,5 & 11,0 & 12,5 \\
\hline Avec expérience MI (2) & 21,3 & 73,1 & 21,4 & 8,1 & 13,0 & 19,4 \\
\hline
\end{tabular}

(1) Ménages avec enfant décohabitant au dénominateur.

(2) Ménages dont au moins un des enfants réside à l'étranger et/ou l'un des membres a effectué une migration internationale.

Source : ENTAR 2001, ENTOL 2002, ENYAC 2002, ENVIL 2002.

Logiquement, les migrations avec retour, comme la résidence des enfants vivant à l'étranger, conduisent à observer la prépondérance de l'espace argentin, mais aussi l'importance non négligeable d'autres pays. Par ailleurs, ces données traduisent des disparités selon le milieu d'habitat. Ainsi, l'expérience migratoire des citadins est plus ouverte sur le continent et le monde, alors que les campagnes sont strictement orientées vers l'Argentine. Une vingtaine d'États appartenant essentiellement au continent américain et à l'Europe sont cités. Les effectifs d'enquête ${ }^{28}$ se révèlent insuffisants pour les répartir mais certaines tendances se profilent. D'après le fichier des migrations, le Brésil et le Chili sont les principales zones de réception; or d'après celui des enfants résidant à l'étranger, il s'agit plutôt des États-Unis et de l'Europe (l'Espagne notamment, n'apparaît qu'ici) ; ce qui peut être significatif d'une orientation émergente. Enfin, les liens migratoires identifiés avec les États-Unis sont quasi exclusivement le fait de la population de Tarija.

Tableau 5 : Répartition des migrations internationales et des enfants résidant à l'étranger selon la destination, par lieu d'enquête

\begin{tabular}{|l|l|l|l|l|}
\hline & \multicolumn{2}{|l|}{ Migrations internationales } & \multicolumn{2}{l|}{ Enfants résidant à l'étranger } \\
\hline Lieu d'enquête & Argentine & Autre & Argentine & Autre \\
\hline Ville de Tarija & 83,5 & 16,5 & 87,9 & 12,1 \\
\hline Vallée de Tolomosa & 99,5 & 0,5 & 100,0 & 0,0 \\
\hline
\end{tabular}




\begin{tabular}{|l|l|l|l|l|}
\hline Yacuiba & 89,9 & 10,1 & 91,2 & 8,8 \\
\hline Villamontes & 82,5 & 17,5 & 93,9 & 6,1 \\
\hline Carapari & 82,4 & 17,6 & (a) & (a) \\
\hline Caigua & 95,0 & 5,0 & (a) & (a) \\
\hline
\end{tabular}

(a) : Effectifs insuffisants.

Source : ENTAR 2001, ENTOL 2002, ENYAC 2002, ENVIL 2002.

\section{Les spécificités locales}

Les indicateurs présentés suggèrent bien qu'en termes de fréquence ou de localisation de la migration, les localités enquêtées se différencient. Sans entrer dans l'analyse monographique qui seule permet de restituer la spécificité de chaque processus (Domenach, 2007), on évoquera par quelques exemples, la nature des déterminants en cause dans les variations observées.

Tableau 6 : Part des migrations en Argentine et des enfants qui résident en Argentine, par lieu d'enquête

\begin{tabular}{|ll|l|l|l|l|l|l|}
\hline Part des... & Tarija & $\begin{array}{l}\text { Vallée } \\
\text { Tolomosa }\end{array}$ & de & Yacuiba & $\begin{array}{l}\text { Villa- } \\
\text { montes }\end{array}$ & Carapari & Caigua \\
\hline $\begin{array}{l}\text { enfants qui résident en } \\
\text { Argentine (1) }\end{array}$ & $18,4 \%$ & $60,8 \%$ & $13,5 \%$ & $5,8 \%$ & $2,5 \%$ & $4,4 \%$ \\
\hline $\begin{array}{l}\text { enfants qui résident en } \\
\text { Argentine (2) }\end{array}$ & $36,2 \%$ & $70,5 \%$ & $23,2 \%$ & $9,6 \%$ & $3,6 \%$ & $6,5 \%$ \\
\hline migrations en Argentine & $18,4 \%$ & $61,8 \%$ & $13,5 \%$ & $5,8 \%$ & $2,5 \%$ & $4,4 \%$ \\
\hline
\end{tabular}

(1) Dénominateur : ensemble des enfants décohabitants.

(2) Dénominateur : enfants décohabitants qui résident dans une localité différente de celle des parents.

Source : ENTAR 2001, ENTOL 2002, ENYAC 2002, ENVIL 2002.

La comparaison de la Vallée de Tolomosa (campagnes traditionnelles) et de la communauté de Caigua (campagnes de colonisation récente) illustre l'incidence de l'accès aux ressources. Clairement, les populations des vallées andines sont depuis longtemps confrontées aux limites du milieu. Le cas de Tolomosa est un exemple de système migratoire spécialisé : on y recense des migrations de travail dans $86 \%$ des cas, en Argentine ( $85 \%$ ), et principalement dans l'agriculture (79\%). Parallèlement, les enfants partis reproduisent ces structures. La proportion extrême de 73 \% de ménages avec expérience de la migration internationale est l'expression de cette adaptation sociale, qui s'inscrit dans une certaine antériorité par rapport à d'autres territoires. Une telle organisation des réseaux migratoires à l'extérieur ne peut exister au même moment à Caigua, où la majorité des hommes sont des immigrants venus travailler 
(83\%) et le cycle de formation du minifundio se trouve moins avancé. Même si la communauté expulse désormais une partie significative de sa population (68\% des enfants décohabitants ne résident pas à Caigua), ils sont le plus souvent (31\%) dans la ville voisine (Villamontes), puis à Tarija (15\%), à Santa Cruz (9\%) et en Argentine dans seulement $7 \%$ des cas; ils étudient $(20 \%)$ et quand ils travaillent $(58 \%)$, sont peu insérés dans l'agriculture (17\%).

De même, dans les petites localités urbaines (Villamontes) ou semi-urbaines (Carapari) orientales, exposées aux effets de l'expansion des activités gazières, la société locale n'a pas lieu d'être focalisée sur l'espace argentin. Parmi les six localités enquêtées, ce sont les populations les moins connectées avec le pays voisin. Finalement, au niveau des populations urbaines, les deux villes principales génèrent les systèmes migratoires les plus orientés vers l'Argentine. Paradoxalement, cette orientation est plus affirmée à Tarija (tableau 6), la ville la plus éloignée de la frontière, ce que l'on imputera en partie à trois déterminants. Tout d'abord, à Yacuiba, la part des migrations vers l'Argentine est atténuée par l'expérience antérieure des non natifs (Souchaud, 2007): avant l'installation à Yacuiba, $20 \%$ des migrations se font en Argentine ; après l'installation, l'effet de frontière joue pleinement et la proportion double. Ensuite, on constate un effet de hiérarchie urbaine. Tarija offrant la plus forte concentration d'emplois dans la région, l'émigration se dirige alors principalement vers les grandes villes du pays (La Paz, Santa Cruz) ou d'Argentine. À l'inverse, les candidats à l'émigration, des autres localités du département, peuvent trouver dans la capitale un échelon urbain satisfaisant. Enfin, Tarija est un pôle d'accueil traditionnel des populations des vallées andines (près de la moitié des non natifs proviennent des secteurs occidentaux du Tarija, du Chuquisaca ou du Cochabamba), qui sont habituées à migrer en Argentine. Ainsi les composantes de l'immigration sont de nature à influer sur l'orientation de l'émigration ${ }^{29}$.

Les différenciations peuvent également être observées en perspective diachronique, par l'estimation du trend des flux migratoires vers l'Argentine ${ }^{30}$ (graphique 2). L'exemple de Tarija et de Yacuiba est révélateur de la répercussion contrastée d'un effet de frontière comme le cycle de l'économie argentine accompli entre 1992 et 2001. Dans la capitale départementale, la distribution des migrations montre des flux peu intenses sur l'essentiel de la période : le nombre des départs dépasse celui des retours dès 1992, la croissance est linéaire jusqu'à l'inflexion de 1999, relayée par une forte concentration des retours. Cette évolution signifie qu'avant même l'effondrement du taux de change, l'Argentine était devenue moins attractive. À Yacuiba, la chronique est sensiblement différente car l'essor de la ville, en pleine prospérité commerciale, interfère avec l'attractivité de l'espace argentin. Au début des années quatre-vingt-dix, les flux de départ vers l'Argentine s'intensifient, puis le mouvement s'inverse et les retours prédominent. Mais la phase de prospérité locale tend à s'épuiser (ce qui est corroboré notamment par les arrivées en provenance de Yacuiba, captées à Tarija et Villamontes), et le regain des départs vers l'Argentine témoigne d'un nouvel arbitrage des populations en faveur de l'extérieur. Les prémisses de la grande crise se traduisent ici par une intensification des départs comme des retours, avec une durée de migration réduite. 
Graphique 2 : Les migrations à destination de l'Argentine des personnes âgées de 15 ans et plus : nombre de départs et de retours selon l'année de fin (1990-2001)
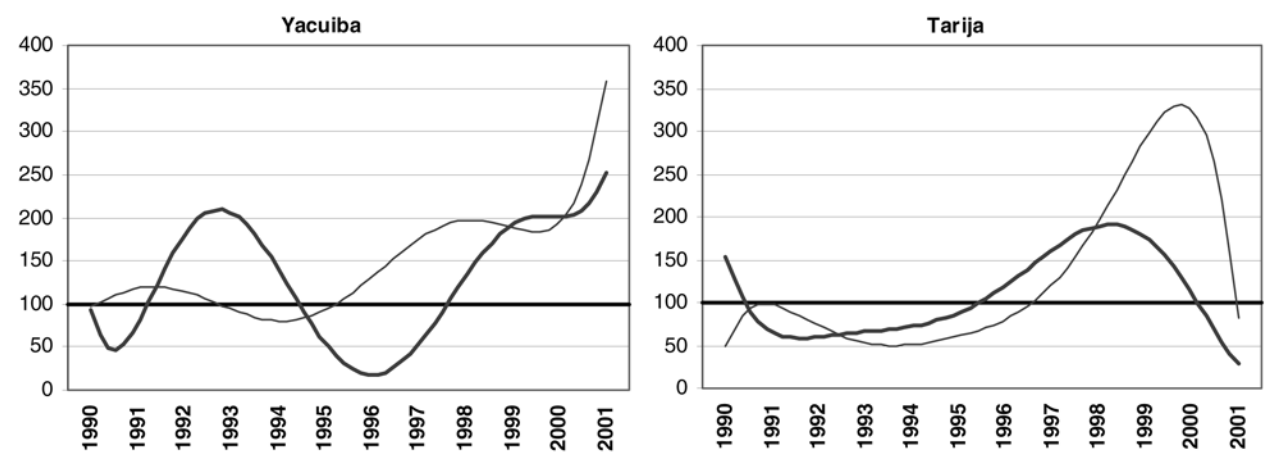

\section{Entre tradition et modernité : le renouvellement des migrations vers les provinces frontalières}

L'observation des destinations migratoires en Argentine (tableaux 7, 8) montre à la fois une cohérence avec les tendances de déplacement du courant migratoire (cf. partie I), et la permanence de territorialités plus traditionnelles. Concernant la désaffection des provinces frontalières, le diagnostic doit être nuancé. Les migrations récentes (1990-2001) les désignent comme lieu de réception de premier ou second plan (33\% des cas à Tarija, $67 \%$ à Tolomosa, $39 \%$ à Yacuiba, $75 \%$ à Villamontes). La migration en cours dont témoigne la localisation des enfants résidant en Argentine corrobore cet ancrage. Mais si l'on constate une diminution globale de la part des migrations à destination des provinces frontalières par rapport aux années antérieures à 1970, seul le cas de Tarija s'inscrit dans le schéma d'une baisse continue. Selon les migrations des habitants de Yacuiba, l'inflexion intervient dans les années quatre-vingt-dix, tandis que les provinces frontalières ont connu un regain d'intérêt la même décennie pour les habitants de Tolomosa (vers la capitale de Jujuy - San Salvador -, le secteur de Fraile Pintado, la lisière du Chaco) et de Villamontes (San Salvador). Néanmoins, la tendance de désaffection de la province de Jujuy est très nette à Tarija et Yacuiba. 
Tableau 7 : Les migrations et les enfants résidant en Argentine selon la destination, par lieu d'enquête (en \%)

\begin{tabular}{|c|c|c|c|c|c|c|c|c|}
\hline \multirow[b]{2}{*}{ Province } & \multicolumn{2}{|c|}{ Tarija } & \multicolumn{2}{|c|}{$\begin{array}{l}\text { Vallée de } \\
\text { Tolomosa }\end{array}$} & \multicolumn{2}{|c|}{ Yacuiba } & \multicolumn{2}{|c|}{ Villamontes } \\
\hline & 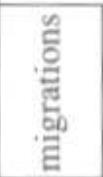 & 总㐬 & 总 & 总善 & 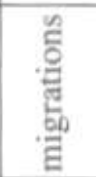 & 总蔍 & 苞 & 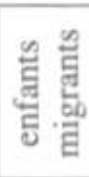 \\
\hline Jujuy & 13,8 & 10,5 & 33,7 & 29,0 & 13,8 & 6,0 & 17,8 & 10,3 \\
\hline Salta & 26,5 & 25,4 & 26,8 & 15,1 & 37,1 & 65,1 & 55,6 & 55,2 \\
\hline Buenos Aires & 40,2 & 44,7 & 16,1 & 21,7 & 29,9 & 20,5 & 11,1 & 13,8 \\
\hline Cordoba, Mendoza, Sta Fé & 14,3 & 16,7 & 13,8 & 18,0 & 12,6 & 7,2 & 8,9 & 20,7 \\
\hline Autre & 5,3 & 2,6 & 9,7 & 16,2 & 6,6 & 1,2 & 6,7 & 0,0 \\
\hline Total provinces frontal. & 40,2 & 36,0 & 60,5 & 44,1 & 50,9 & 71,1 & 73,3 & 65,5 \\
\hline Total & 100,0 & 100,0 & 100,0 & 100,0 & 100,0 & 100,0 & 100,0 & 100,0 \\
\hline
\end{tabular}

Source : ENTAR 2001, ENTOL 2002, ENYAC 2002, ENVIL 2002.

Concernant l'établissement du courant migratoire dans le reste du pays, on constate depuis Tarija et Yacuiba, l'affirmation de la destination province de Buenos Aires, avec la spécificité dans le premier cas, d'une forte concentration dans le Grand Buenos Aires (29\% des enfants résidant en Argentine). Les grandes provinces du centre (Mendoza, Cordoba, Santa Fé) ont été plus particulièrement attractives durant les années 1970-1980 ; Cordoba et Mendoza sont les principales localités citées. Les habitants de Tolomosa ont la particularité d'avoir développé un champ migratoire avec la province de Corrientes (14\% des enfants en Argentine). En définitive, la population de Tarija est la plus en phase avec la tendance générale de localisation des Boliviens en Argentine, tout en restant liée aux provinces frontalières. Les habitants de la vallée de Tolomosa indiquent au contraire une forte singularité, caractérisée par une grande dispersion géographique et la prééminence de la province de jujuy. Enfin, les localités orientales se situent dans un schéma intermédiaire, marqué par une connexion ancienne avec la province de Salta (villes de Salta, Oran et Tartagal). 
Tableau 8 : Les migrations en Argentine selon la période de retour et la destination, par lieu d'enquête (en \%)

\begin{tabular}{|c|c|c|c|c|c|c|c|c|c|c|c|c|c|c|c|c|}
\hline & \multicolumn{4}{|c|}{ Tarija } & \multicolumn{4}{|c|}{ Tolomosa } & \multicolumn{4}{|c|}{ Yacuiba } & \multicolumn{4}{|c|}{ Villamontes } \\
\hline Destination & 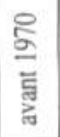 & $\frac{\stackrel{2}{\circ}}{\frac{\alpha}{\delta}}$ & $\begin{array}{l}\overline{8} \\
\text { స్ } \\
\text { ठे. }\end{array}$ & $\stackrel{\Xi}{\mathrm{g}}$ & 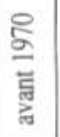 & $\frac{\frac{2}{2}}{\frac{\partial}{2}}$ & $\begin{array}{l}\bar{్} \\
\text { ते } \\
\text { ठे. }\end{array}$ & 평 & 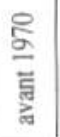 & 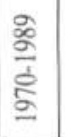 & 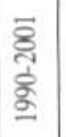 & ฐ & 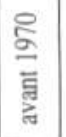 & $\frac{\frac{2}{2}}{\frac{\delta}{\sigma}}$ & $\begin{array}{l}\overline{8} \\
\text { స్. } \\
\text { \&. }\end{array}$ & 졍 \\
\hline Jujuy & 35,3 & 14,9 & 5.1 & 13,8 & 41,9 & 19,3 & 38,2 & 33,7 & 10,5 & 23,9 & 5,1 & 13,8 & (a) & (a) & (a) & 17,8 \\
\hline Salta & 29,4 & 22,4 & 28,2 & 26,5 & 23,3 & 22,7 & 29,0 & 26,8 & 47,4 & 38,8 & 34,2 & 37,1 & (a) & (a) & (a) & 55,6 \\
\hline Buenos Aires & 23,5 & 37,3 & 50,0 & 40,2 & 7,0 & 22,7 & 14,9 & 16,1 & 15,8 & 22,4 & 39,2 & 29.9 & (a) & (a) & (a) & 11,1 \\
\hline Cordoba, Mendoza, Sta Fé & 11,8 & 19,4 & 9,0 & 14,3 & 14,0 & 26,1 & 8,7 & 13,8 & 15,8 & 9,0 & 15,2 & 12,6 & (a) & (a) & (a) & 8,9 \\
\hline Autre & 0,0 & 6,0 & 7,7 & 5,3 & 14,0 & 9,1 & 9,1 & 9,7 & 10,5 & 6,0 & 6,3 & 6,6 & (a) & (a) & (a) & 6,7 \\
\hline Total provinces frontalières & 64,7 & 37,3 & 33,3 & 40,2 & 65,1 & 42,0 & 67,2 & 60,5 & 57,9 & 62,7 & 39,2 & 50,9 & 92,3 & 57,9 & 75,0 & 73,3 \\
\hline Total & 100,0 & 100,0 & 100,0 & 100,0 & 100,0 & 100,0 & 100,0 & 100,0 & 100,0 & 100,0 & 100,0 & 100,0 & 100,0 & 100,0 & 100,0 & 100,0 \\
\hline
\end{tabular}

(a) Effectifs insuffisants

Source : ENTAR 2001, ENTOL2002, ENYAC 2002, ENVIL2002.

\section{Conclusion}

41 À l'échelle du système migratoire Bolivie-Argentine, le Tarija reste un petit bassin d'expulsion, comparé aux masses provenant de départements comme le Potosi ou le Cochabamba. Toutefois, la croissance rapide de la population crée un phénomène de densification démographique inédit sur cette frontière, qui alimente le développement des mobilités spatiales à destination de l'Argentine. Car en dépit des cycles qui stimulent la valorisation de l'espace et son peuplement, l'émigration est omniprésente. D'autant que les phases d'essor sont souvent suivies d'une phase dépressive symétrique. Terre d'accueil, le Tarija est aussi un espace que l'on quitte massivement, depuis les campagnes andines traditionnellement, mais aussi depuis la capitale et Yacuiba, qui, malgré leur dynamisme démographique, pourvoient largement aux cohortes de jeunes partis vivre en Argentine.

Historiquement, l'Argentine est la destination de prédilection des migrants internationaux boliviens, et c'est naturellement aussi le cas des populations frontalières de ce pays. Toutefois, la question qui se pose maintenant, au regard de la réorientation du système migratoire bolivien, est de savoir si l'intégration d'une périphérie comme le Tarija va se répercuter sur ses connexions transnationales. Les enquêtes ont été réalisées trop tôt pour l'illustrer, mais il est probable que le changement émergera des plus grandes villes. On constate d'ores et déjà que Tarija est la plus cosmopolite par ses réseaux migratoires (États-Unis, Europe), alors que l'expérience internationale des populations rurales se limite à l'Argentine. À moins que l'étonnante capacité d'innovation du paysannat andin lui permette de trouver de nouvelles niches dans un espace international non balisé. Le département du Tarija reste dans les circonstances actuelles un terrain pertinent d'observation des migrations internationales, y compris pour étudier ces nouveaux phénomènes.

La perte d'attractivité des provinces frontalières, berceau du courant migratoire bolivien en Argentine, ne se vérifie que partiellement. Effectivement, l'évolution de la 
géographie des migrations témoigne bien à Yacuiba et Tarija, d'un report vers les grandes villes et en particulier Buenos Aires. Car la population des strates supérieures de l'armature urbaine tend à inscrire ses mobilités dans le réseau des métropoles, ce que l'on constate à la fois pour les migrations internes et externes. Les petites villes comme Villamontes révèlent des champs migratoires nettement portés sur les villes moyennes argentines proches et Salta. Du côté des campagnes andines, la région frontalière prime aussi, mais la référence aux systèmes agro-industriels du tabac et de la canne à sucre est révolue. Les paysans chapacos œuvrent dans le maraîchage, valorisant les vallées chaudes de Salta et Jujuy, au lieu de celles du département, faute d'accès au foncier.

Cet aspect des différenciations entre lieux d'enquête illustre bien la fragilité des agrégats spatiaux utilisés dans les analyses macro. L'analyse du développement des courants migratoires doit s'enrichir de la connaissance de la géographie des pays de départ. Le cas du Tarija montre que les départements boliviens, mêmes petits, ne sauraient constituer une catégorie homogène, a fortiori ceux positionnés à l'articulation des Andes et des terres basses. Le principal effet de frontière attendu - la surreprésentation des migrations en Argentine - passe avant tout par le prisme du territoire. L'ancienneté du peuplement, les structures agraires, l'éloignement de la frontière, l'origine des immigrants, la position de la localité dans la hiérarchie urbaine, entre autres exemples, conditionnent les territorialités et les modalités du recours à l'espace argentin. En définitive, l'approche comparative développée offre un premier aperçu des processus migratoires liés à la recomposition régionale du Sud bolivien. Elle appelle l'étude spécifique des localités enquêtées dans le Tarija, qui constituent autant de micro-systèmes migratoires pouvant éclairer singulièrement le phénomène des migrations vers l'Argentine.

\section{BIBLIOGRAPHIE}

ANDREU Domingo, MARTINEZ Rosana (2006) La población latinoamericana censada en España en 2001 : un retrato sociodemográfico, Notas de población n 81, CEPAL, pp. 99-127.

BENENCIA Roberto, KARASIK Gabriela (1995) Inmigración limítrofe : los bolivianos en Buenos Aires, Biblioteca Política Argentina, Centro Editor de América Latina, Buenos Aires.

BOLOGNA Eduardo (2005) La reversibilidad en los estadios avanzados de los sistemas migratorios, el caso de Argentina y Bolivia, thèse de doctorat en démographie, Facultad de ciencias económicas Universidad Nacional de Córdoba-Université Paris 5, Sorbonne, 443 p.

CODEPO (2003) La Bolivia del futuro y sus implicaciones demográficas para el desarrollo, Consejo de Población para el Desarrollo sostenible, La Paz, 81 p.

CORTES Geneviève (1995) La migration : survie et mutations des sociétés paysannes andines. Deux exemples dans le Valle Alto de Cochabamba (Bolivie), thèse de doctorat de $3^{\mathrm{e}}$ cycle, Université de Toulouse Le Mirail, 521 p. 
CORTES Geneviève (1995) L'émigration rurale dans les vallées inter-andines de Bolivie, Revue Européenne des Migrations Internationales (11) 2, 1995, pp. 113-129.

D'ANDREA Nicolas (2004) Effets de frontière, migrations et redistribution du peuplement en Bolivie : les dynamiques territoriales dans le département du Tarija, thèse pour obtenir le grade de docteur en « aménagement de l'espace, urbanisme », Université Aix-Marseille III, Institut d'Aménagement Régional, $424 \mathrm{p}$.

D'ANDREA Nicolas, MARTIN Ceydric (2007) Análisis de los factores de estabilidad del crecimiento demográfico de la ciudad de Tarija, in DOMENACH Hervé, CELTON Dora, ARZE Hugo, HAMELIN Philippe (Ed. sc.), (2007) Movilidad y procesos migratorios en el espacio de frontera argentino-boliviana, Editions Copiar, Cordoba (Argentine), 307 p.

DELLA PAOLERA G. (entretien avec) (2006) Argentine quel avenir ? Géopolitique, n 96, octobre 2006, pp. 34-38.

DOMENACH Hervé, CELTON Dora, ARZE Hugo, HAMELIN Philippe (Ed. sc.) (2007) Movilidad y procesos migratorios en el espacio de frontera argentino-boliviana, Éditions Copiar, Cordoba (Argentine), $307 \mathrm{p}$.

DOMENACH Hervé, CELTON Dora et al (1995) La comunidad boliviana en Córdoba. Caracterización y proceso migratorio, ORSTOM, UNC, LPE.

INDEC (1996) La población no nativa de la Argentina 1869-1991, serie análisis demográfico, 6, elaborado por Guillermo Maccío y Diva Elizalde, Buenos Aires.

KARASIK Gabriela, BENENCIA Roberto (1999) Apuntes sobre la migración fronteriza. Trabajadores bolivianos en Jujuy, Estudios migratorios latinoamericanos, año 13-14, n 40-41, pp. 569-594

LABROUSSE Alain (2002) Article « Bolivie », Atlas géopolitique des drogues, De Boeck Université, Louvain-la-Neuve, pp. 111-118.

LANGER Erick (1990) «Introducción », Andes, n 1, Salta, pp. 7-10.

MARTIN Ceydric (2005), Complexe migratoire et distribution spatiale de population dans le Sud bolivien. Enquêtes en milieu rural et urbain dans le département du Tarija, Thèse de doctorat de démographie, Paris, Université Paris 5, Faculté des sciences humaines et sociales - Sorbonne, $703 \mathrm{p}$.

RENARD Jean-Pierre (1992) Populations et frontières : problématiques et méthodes, Espace, populations, sociétés, 1992-2, pp. 167-184.

RIVIERE D’ARC Hélène (1979) Espace national et périphéries frontalières en Bolivie, Problèmes d'Amérique latine, p. 117.

ROCA José-Luis (1999) Fisionomía del regionalismo boliviano, Plural, La Paz, 350 p.

SOUCHAUD Sylvain (2007) Yacuiba Islote de la mundialisación, in DOMENACH Hervé, CELTON Dora, ARZE Hugo, HAMELIN Philippe (Ed. sc.), (2007) Movilidad y procesos migratorios en el espacio de frontera argentino-boliviana, Éditions Copiar, Cordoba (Argentine), 307 p.

WHITEFORD Scott (1981) Workers from the North. Plantations, Bolivian labor and the City in the Northwest Argentina, University of Texas Press, Austin. 


\section{NOTES}

1. En classant les provinces de part et d'autre d'une courbe suivant l'inflexion des Andes à hauteur du massif subandin, on constate que le rapport d'effectifs Orient-Occident était de 1 pour 9 en 1950 ; cinquante ans plus tard, il est sensiblement égal à 1 pour 1,4. (d'Andréa, 2004).

2. Entre 1950 et 2001 , la population a été multipliée par 2,7 pour une variation nette de 5,5 millions d'habitants, qui ont porté l'effectif à 8,3 millions d'habitants. Le taux d'accroissement naturel aurait été de nature constante sur la période, autour de 2,4 \% annuels (CELADE). Il aurait alors commencé à décliner vers l'an 2000 et selon une hypothèse moyenne (CODEPO, 2003), le pays devrait gagner un million d'individus tous les 5 ans jusqu'en 2020, où il atteindra approximativement plus de 12 millions d'habitants.

3. Source : Instituto Nacional de Estadística (INE).

4. Source: base de données Investigación de la Migración Internacional en América Latina (IMILA). Cette source produit une matrice des migrations sur durée de vie, croisant lieu de naissance et lieu de résidence pour tous les pays d'Amérique, par décennie de recensement (sans ajustement). L'outil offre une approximation de l'évolution de la concentration des stocks de migrants internationaux par pays.

5. À titre de comparaison, l'Argentine accueillait au début des années $90: 88 \%$ des natifs du Paraguay, 69 \% des natifs de l'Uruguay, $61 \%$ des natifs du Chili, $12 \%$ des natifs du Brésil, $7 \%$ des natifs du Pérou.

6. Il s'agit des EPH (encuesta población y hogar) réalisées par l'Instituto Nacional de Estadísticas y Censos (INDEC), enquêtes-ménages appliquées sur les principales aires urbaines du pays. La comparaison porte sur les collectes d'octobre 2000 et octobre 2002. Il est à noter premièrement que la variable migratoire retenue pour l'analyse est un agrégat de tous les natifs de pays limitrophes plus le Pérou. Deuxièmement, les analyses n'ont pu être réalisées qu'en valeur relative, pour cause de modification de la méthode d'échantillonnage, aucun chiffrage de la variation nette des populations n'a pu ainsi être réalisé. Troisièmement, l'interprétation est délicate car la population qui serait sortie de l'univers enquêté (les principales aires urbaines), n'est pas nécessairement sortie du pays, elle peut se trouver en partie dans des secteurs ruraux ou des petites villes.

7. C'est ce qui ressort de diverses enquêtes, des registres d'entrées et de sortie, ainsi que des recensements prenant en compte la migration internationale (Colombie, 2005 ; Équateur, 2001 ; Pérou, encuesta continua 2006). Toutefois, les stocks de migrants accumulés aux États-Unis dépassent encore nettement les implantations en Europe. Par exemple, le Dane (institut de statistiques colombien) évalue la part de ses ressortissants vivant à l'extérieur en 2005 pour $35 \%$ aux États-Unis, 23 \% en Espagne, 20 \% au Venezuela, puis $3 \%$ en Équateur. (DANE, Boletín Censo General 2005, Perfil Colombia).

8. D’après le Padrón Municipal, le registre administratif actualisé dans lequel doivent s'inscrire toutes les personnes qui vivent habituellement en Espagne, il y avait 2117 résidents boliviens en 2000, 6619 en 2001, 13517 en 2002, 28432 en 2003, 52345 en 2004, 97945 en 2005 et 139802 en 2006.

9. L'Encuesta Complementaria de Migraciones Internacionales (ECMI) est une série d'enquêtes spécialisées réalisées par l'INDEC, en utilisant le recensement de 2001 comme base de sondage. Le questionnaire permet de renseigner les caractéristiques socio-démographiques des migrants et des ménages auxquelles ils appartiennent, avec une attention spécifique aux paramètres de la migration et des liens avec le pays d'origine. L'enquête a été administrée dans les principales concentrations de référence, pour les populations étrangères provenant des pays limitrophes et du Pérou. Concernant la population bolivienne, la collecte a été effectuée dans le Grand Buenos Aires, le Grand Jujuy et le Grand Salta. 
10. Les tableaux publiés pour l'enquête de Buenos Aires distinguent comme lieu de première résidence : le Grand Buenos Aires, le reste de la province de Buenos Aires, la province de Jujuy, les autres provinces.

11. Selon l'ECMI, les natifs de Bolivie enquêtés dans le Grand Buenos Aires ont dans $47 \%$ des cas connu trois résidences ou plus quand leur arrivée en Argentine est antérieure à 1970; la proportion est de $28 \%$ pour les migrants arrivés dans les années 1970-1979, $21 \%$ dans les années 1980-1989, et seulement $10 \%$ pour la période 1990-2003. Voir aussi le cas de la communauté bolivienne de Neuquén analysé par E. Bologna (2004).

12. Hors intérieur de la plaine du Chaco, de tendance aride à semi-aride.

13. Nom de la population de langue espagnole, née du métissage des Espagnols installés dans ces vallées depuis 1539 et des tribus du groupe Chichas qui peuplaient déjà la zone.

14. En 1543, la Couronne espagnole fonde la Vice-royauté du Pérou pour administrer le Sud de l'Empire, soit une juridiction comprenant alors toute la zone conquise de Panama à l'estuaire de La Plata.

15. Il s'agit d'un couloir de circulation altiplanique reliant $\mathrm{La} \mathrm{Paz}$, à la province de Jujuy (Argentine), via la quebrada de Humahuaca. L'intersection se produit à $3400 \mathrm{~m}$ d'altitude, engendrant un doublet urbain - La Quiaca côté argentin et Villazon côté bolivien, dont le caractère stratégique s'est affirmé durant le XIX siècle, avec la stabilisation de la frontière et la connexion des réseaux ferrés boliviens et argentins (la ligne Atocha-Villazon est inaugurée en 1925).

16. Cet axe est tout d'abord consolidé par la voie ferrée Santa Cruz-Yacuiba terminée à la fin des années 50, puis par des travaux routiers. Le revêtement complet de la voirie a été réalisé récemment (2002), constituant la seule route dans le sens Nord-Sud à offrir cet avantage. La zone occidentale (axe La Paz-Potosi-Villazon) conserve encore un réseau mixte comportant de longs tronçons de pistes de qualité exécrable.

17. Entre 1950 et 2001, la population du pays a augmenté selon un taux d'accroissement annuel moyen de 2,0 \%, contre 2,2 \% dans le Tarija, 3,9\% dans le Santa Cruz, 1,8\% dans le département de La Paz ou encore $0,86 \%$ dans celui du Potosi.

18. En 2004, a été enquêtée la ville de Bermejo, mais nous ne commenterons pas ici les résultats de cette enquête, décalée dans le temps.

19. La réfection de la route au début des années 2000 a complètement modifié ce paramètre. Le temps de déplacement peut aujourd'hui être réduit à deux heures trente en voiture.

20. En effet, Tarija est un lieu de réception traditionnel des migrations rurales de la zone andine méridionale (départements du Chuquisaca et du Tarija). Dans les années 80, la ville accueille des flux de migrants issus des bassins miniers sinistrés (département du Potosi principalement, d'Oruro et de La Paz). Enfin dans les années 90, s'ajoutent les apports migratoires des sub-régions orientales du département, notamment du fait de la crise des villes-frontière.

21. Il est intéressant de noter que cette activité devrait être relancée dans le contexte économique actuel, favorable aux cultures industrielles et pouvant être financé par la rente gazière.

22. Le statut résidentiel de la descendance des chefs de ménage donne par exemple un aperçu de la sélectivité de l'émigration. Il apparaît ainsi que le lieu de résidence des descendants est corrélé à la taille de l'exploitation: si le ménage de référence est non producteur, $70 \%$ des enfants résident à l'extérieur de la communauté ; 56 \% s'il s'agit d'une micro-exploitation ; $37 \%$ dans le cas d'une petite exploitation.

23. L'économie de la drogue a connu un pic de prospérité au début des années 80 . L'intervention américaine de 1986 marque le début de la militarisation du Chaparé et de l'éradication de la coca mais l'activité prospère jusqu'aux alentours de 1994, si l'on se réfère aux statistiques du Département d'Etat américain. Toutefois, malgré la réduction drastique des superficies de cocaïers illicites, le rôle de la Bolivie semble dès lors se reporter sur la fabrication du produit fini, 
le chlorydrate de cocaïne, exporté essentiellement via le Brésil. Voir G.Cortes (1995), A. Labrousse (2002).

24. El blanqueo de dinero en Bolivia, interview de Benjamín Grossman Parrondo, in Temas en la crisis, año XVII - n 52. I-98, p. 25.

25. Le code du pétrole de 1938 fixe les royalties à $11 \%$ de la production départementale. Mais les premières redevances sont effectivement reversées aux départements à partir de 1957. Cf. J.-L Roca, 1999.

26. $87 \%$ du gaz et $83 \%$ du pétrole et condensé (réserves prouvées et probables au $1^{\mathrm{er}}$ janvier 2003, selon le ministère des mines et des hydrocarbures).

27. NB : si chaque ménage est composé d'une ou plusieurs personnes ayant pu effectuer une migration, tous les ménages n'ont pas forcément d'enfants décohabitants. De fait, en plaçant la population des ménages avec enfant décohabitant au dénominateur, la "prévalence » de l'émigration internationale s'accentue.

28. Concernant les pays autres que l'Argentine, les enquêtes comprennent au total 75 cas de migrations avec retour (dont 40 dans la population enquêtée à Tarija) et 29 lieux de résidence actuels pour les enfants des chefs de ménage. À titre indicatif, apparaissent par ordre d'occurrence, toutes localités confondues sans pondération: Brésil, Chili, États-Unis, Équateur, Paraguay, Russie, Mexique, Venezuela, Nicaragua, Belgique, Japon, Pérou, Allemagne, Cuba, Honduras, Italie, Colombie, Roumanie, Autriche, Uruguay.

29. En moyenne, $26 \%$ des émigrants se dirigent vers l'Argentine, la proportion est de $44 \%$ quand le chef de ménage de référence n'est pas natif de la ville mais d'une autre localité de la zone occidentale du département, du Chuquisaca ou du Cochabamba (C. Martin, 2005).

30. L'enquête de Tarija a eu lieu en septembre 2001 et celle de Yacuiba en mars 2002, la fin de la série n'est donc pas strictement comparable. Dans le cas de Tarija, l'année 2001 étant diminuée de près de quatre mois, le nombre des retours l'est aussi, ce qui explique en partie la plongée de la courbe. De même, la possibilité d'enregistrer des départs pour l'année 2001 est quasi nulle puisqu'il faut que la personne ait été absente au moins six mois et soit aussi revenue.

\section{RÉSUMÉS}

Dans le contexte de mutation de l'espace bolivien, certaines périphéries frontalières ont connu ces cinquante dernières années un essor inédit. L'article présente le cas du département du Tarija, qui bouleverse la configuration du peuplement à la frontière de l'Argentine. Se pose alors la question de l'insertion de cette population frontalière dans le système migratoire BolivieArgentine. L'article caractérise la dynamique spatiale du courant migratoire bolivien en Argentine et montre à partir de sources censitaires, que l'attractivité des provinces frontalières s'est progressivement réduite, excepté pour les habitants du Sud bolivien. Puis il identifie au travers d'enquêtes socio-démographiques, les dynamiques territoriales qui conditionnent le rapport spécifique des tarijéniens, à l'espace argentin : commerce transfrontalier, exploitation gazière, explosion urbaine, colonisation agricole, crise des campagnes andines.

Regional Recomposition in Southern Bolivia and Migrations to Argentina. In the last fifty years, the geography of Bolivia has been changing, some areas of the frontier periphery have gone through rapid and unprecedented development. This article presents the case of Tarija, the department where settlement at the boundaries of Argentina has been entirely transformed. 
Thus what is the role of border zone inhabitants in Bolivia-Argentina migration system? The article describes the spatial dynamics of Bolivian migratory flow in Argentina. The study of censual sources shows that frontier areas gradually became less and less attractive, all except that of Southern Bolivia. Demographic surveys were conducted and underline that territorial dynamics are a determining factor in the Tarijan's special relationship with Argentina: crossborder trade, urban explosion, agricultural colonization, Andean agriculture crisis.

Recomposición regional en el Sur boliviano y migraciones en Argentina. En el contexto de la mutación del espacio boliviano, algunas periferias fronterizas han conocido un auge inédito en las últimas décadas. El artículo presenta el caso del departamento de Tarija, el cual está transformando el ordenamiento del poblamiento en la frontera con Argentina. Se plantea entonces el tema de la inserción de esta población fronteriza con respecto al sistema migratorio Bolivia-Argentina. Primero, se caracteriza la dinámica espacial de la corriente migratoria de los Bolivianos en Argentina y se muestra con fuentes censales, que la atracción de las provincias fronterizas se redujo paulatinamente, excepto en el caso de los pobladores del sur de Bolivia. Luego se identifican con encuestas socio-demográficas las dinámicas territoriales que condicionan la relación específica de los Tarijeños con el espacio argentino : comercio fronterizo, explotación del gaz, crecimiento urbano, colonización agrícola, crisis en las campos andinos.

\section{AUTEUR}

\section{NICOLAS D'ANDRÉA}

Docteur en Aménagement, Urbanisme (Institut d'Aménagement Régional, Université AixMarseille III). 